\title{
NUMERICAL AND EXPERIMENTAL INVESTIGATION OF WAVE DYNAMIC PROCESSES IN HIGH-SPEED TRAIN/TUNNELS*
}

\author{
Jiang Zonglin (姜宗林) $^{1} \quad$ K. Matsuoka ${ }^{2} \quad$ A. Sasoh ${ }^{2} \quad$ K. Takayama ${ }^{2}$ \\ ${ }^{1}$ (LHD, Institute of Mechanics, Chinese Academy of Sciences, Beijing 100080, China) \\ ${ }^{2}$ (Shock Wave Research Center, Institute of Fluid Science, Tohoku University, 2-1-1 Katahira, \\ Aoba-ku, Sendai 980-8577, Japan)
}

\begin{abstract}
Numerical and experimental investigation on wave dynamic processes induced by high-speed trains entering railway tunnels are presented. Experiments were conducted by using a 1:250 scaled train-tunnel simulator. Numerical simulations were carried out by solving the axisymmetric Euler equations with the dispersioncontrolled scheme implemented with moving boundary conditions. Pressure histories at various positions inside the train-tunnel simulator at different distance measured from the entrance of the simulator are recorded both numerically and experimentally, and then compared with each other for two train speeds. After the validation of nonlinear wave phenomena, detailed numerical simulations were then conducted to account for the generation of compression waves near the entrance, the propagation of these waves along the train tunnel, and their gradual development into a weak shock wave. Four wave dynamic processes observed are interpreted by combining numerical results with experiments. They are: high-speed trains moving over a free terrain before entering railway tunnels; the abrupt-entering of high-speed trains into railway tunnels; the abrupt-entering of the tail of high-speed trains into railway tunnels; and the interaction of compression and expansion waves ahead of high-speed trains. The effects of train-tunnel configurations, such as the train length and the train-tunnel blockage ratio, on these wave processes have been investigated as well.
\end{abstract}

KEY WORDS: high-speed train, tunnel sonic boom, numerical simulation, experiments

\section{INTRODUCTION}

Transportation systems have indeed been improving with the development of modern societies. Among these systems, the high-speed train network has been developed rapidly in several countries in the world during the last three decades. Recent efforts in the establishment of high-speed train network systems are concentrated not only on the economical benefit but also on the possible increase of train speed. For example, the speed of these trains in operation in Japan nowadays has been increased to a level very close to $300 \mathrm{~km} / \mathrm{h}$, and are expected to reach $400 \mathrm{~km} / \mathrm{h}$ every soon, and even faster for magnetic levitation trains in a near future. Trains moving at such a high speed could cause substantial aerodynamic 
problems like what once happened to supersonic airplanes. One of these problems is the so-called "tunnel sonic boom" which occurs at the railway tunnel exit due to the discharge of the waves driven by the train moving in the railway tunnel.

When a high-speed train is moving over a free terrain, compression waves generated in front of the train are quickly attenuated as they propagate away from it. In contrast, in a railway tunnel, those waves are attenuated very slowly. This phenomenon is similar to a shock wave driven by a piston moving in a tube with a constant cross-sectional area because the shock wave is not spatially diverging. The problem is schematically shown in Fig.1. When a high-speed train enters a railway tunnel, a series of compression waves are generated in front of the train and propagate forward at the local sound speed (see Figs.1(a) and 1(b)). These waves develop into a shock wave, usually very weak as shown in Fig.1(c), if the railway tunnel is long enough. Once the shock wave discharges from the tunnel exit, it turns into a blast wave as shown in Fig.1(d). This blast wave may not be physically dangerous, but it is as loud as sonic booms created by supersonic airplanes when the train speed is over $200 \mathrm{~km} / \mathrm{h}$. Expansion waves generated behind the train in Fig.1(b) and the ones induced at the tunnel exit due to the shock wave discharge (see Fig.1(d)) also propagate into the railway tunnel. Interaction of these waves could induce pressure fluctuations that may lead to an uncomfortable environment in passenger cars.

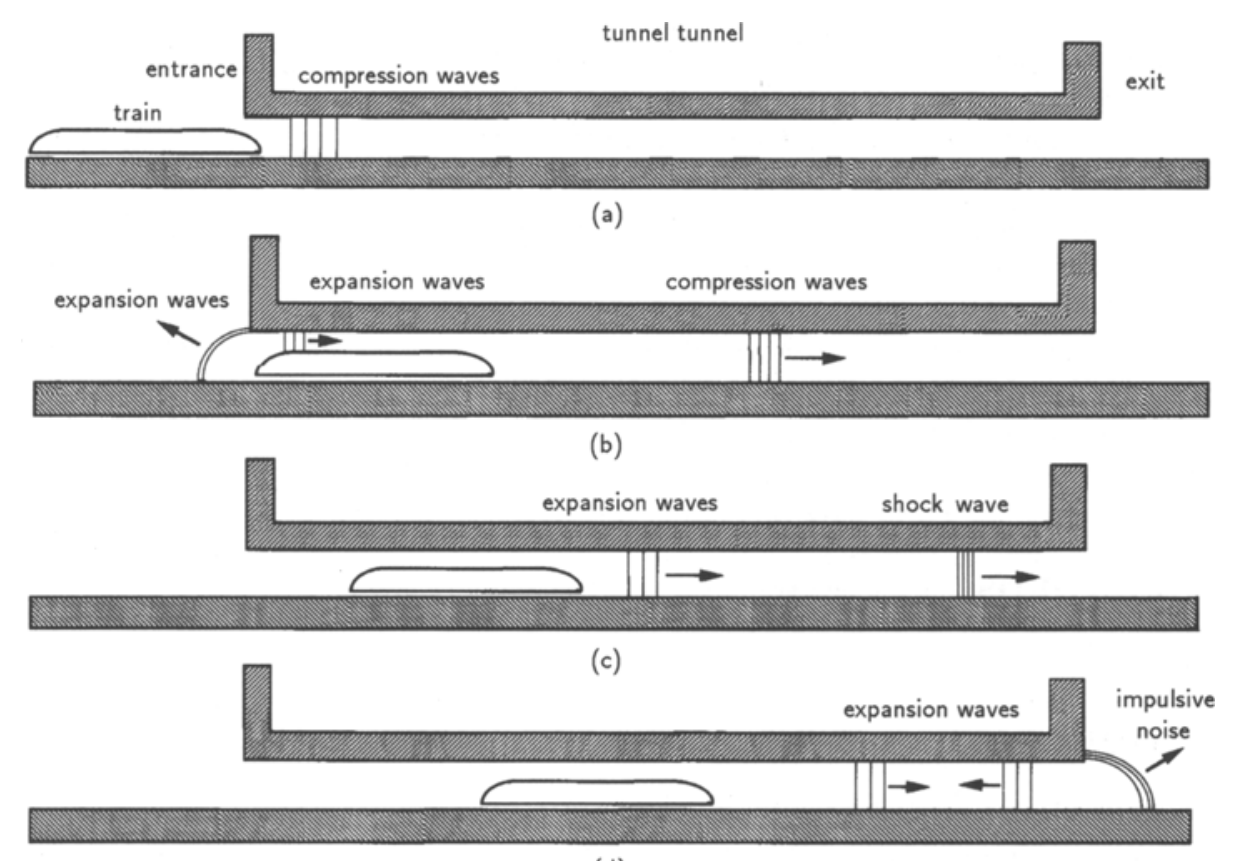

(d)

Fig.1 Schematic diagram for development of train-tunnel sonic booms

The primary generation and attenuation mechanism of tunnel sonic booms is related with train-tunnel configurations, train speed, wave reflection, vortex/wave interaction and viscous effect. The problem has been analyzed previously by $\mathrm{Hara}^{[1]}$, Ozawa ${ }^{[2,3]}$ and Sajben ${ }^{[4]}$ using an acoustic theory to predict the behavior of tunnel sonic booms in train tunnels and subway systems. Such sonic booms were not an important issue in the past 
because the train speed was not so high. However, when a train moving at a speed exceeding about $200 \mathrm{~km} / \mathrm{h}$, like the Japanese Shinkansen in operation nowadays, enters a railway tunnel, compression waves generated in front of the train will coalesce into a weak shock wave $^{[5 \sim 7]}$. In the case of the 0.2 train-tunnel blockage ratio and the $360 \mathrm{~km} / \mathrm{h}$ train speed, the post-shock overpressure is measured to be up to $5 \mathrm{kPa}$. The resulting sonic boom is of the level of $60 \mathrm{~dB}$. The noise in such a level could affect human eardrums because of its high frequencies. Therefore, this problem must be considered in designing the future high-speed train system where the train speed is expected to be much higher.

Matsuo and Aoki ${ }^{[5]}$ had numerically studied the pressure wave propagation in railway tunnels, and the tunnel flow was modelled as one-dimensional and compressible one in their study. Ogawa and Fujii ${ }^{[8]}$ modelled the problem as a two-dimensional inviscid compressible flow and the Euler equations were solved using a zonal method that can treat a moving grid configuration. Some experimental work was conducted by Sasoh et al. ${ }^{[6]}$, Takayama et al. ${ }^{[9]}$ and Sasoh et al. ${ }^{[10]}$ in a scaled train-tunnel simulator and shock tubes. In their work, the weak shock wave developing from compression waves was visualized with holographic interferometry. Various hoods installed at the train-tunnel entrance and porous walls lined inside train tunnels were also investigated as efficient ways to reduce the level of tunnel sonic booms.

Train-tunnel flows driven by a high-speed train in a railway tunnel are turbulent, unsteady and truly three-dimensional. An either numerical or experimental analysis of tunnel sonic booms related to such flows is still a challenging topic. However, if limited only to generation and propagation of compression waves inside a railway tunnel, the problem could be simplified greatly because viscosity effects on the wave propagation are small in the short duration so that the governing equations could be approximated with the Euler equations.

However, in order to simulate wave interactions that result in tunnel sonic booms, at least, the following four physical processes must be taken into account, which could interact with each other and affect tunnel sonic booms in different ways. The first process is the wave propagation induced by a high-speed train moving over a free terrain, which is an analogy of the flow before the train approaches the entrance of a railway tunnel. Compression waves generated in front of the train are attenuated quickly due to their spatial divergence, therefore, their effects on the far-flowfield are small. However, the air in front of and nearby the train is forced to move with the train. This part of the air flow is called the train-affected area.

The second process is a transient one that starts from the moment when a train approaches the tunnel entrance. Compression waves created at this moment in the tunnel propagate not divergently but mainly in one-direction. These waves are similar to those driven by a piston moving in a tube of a diameter of the train-affected area. These waves are sometimes called the precursor compression waves, which induce a sharp pressure rise in the air ahead of the train. This process terminates when the tunnel flow reaches the same state as established by an "infinitely" long train moving in a railway tunnel. The period could be identified by checking the appearance of a constant overpressure rise following a pressure peak in the precursor compression waves.

The third process is related to the abrupt-entering of the train rear into a railway tunnel. A series of expansion waves are generated behind the train, and propagate both 
upstream and downstream (in the train-moving direction). The downstream-propagating waves can catch up with the train and then overtake it since the waves travel at the local sound speed. These waves reduce the overpressure created by the precursor compression waves.

The last process is the flow motion induced by a high-speed train moving in a infinitely long railway tunnel at a constant speed after both compression and expansion waves propagate far ahead of the train. The flowfield could be treated as a steady-state one in train-fixed coordinates. Overpressure generated in front of the train is mainly related to the train-tunnel blockage ratio.

Although some of those four processes have been discussed more or less in literature, as mentioned above, the interaction mechanism of the four wave processes remains still unclear. Both experimental and numerical simulations are conducted to study these wave processes in the present paper. A 1:250 scaled train-tunnel simulator in the Shock Wave Research Center, Tohoku University, was used in the experiment. Numerical simulations were carried out by solving the axisymmetric Euler equations with the dispersion-controlled scheme implemented with moving boundary conditions. Pressure histories at various positions inside the train-tunnel simulator were recorded for two train speeds. The results are used to reveal characteristics of wave interactions in the train-tunnel simulator as well as to validate numerical solutions. After good agreement between the numerical results and the experimental data was achieved more detailed numerical simulations were conducted by varying train speeds, train lengths, and train-tunnel blockage ratio. From these results, the compression-wave generation near the entrance, their transformation into a weak shock wave, the expansion wave propagation and the effects of train-tunnel configurations are discussed for a better understanding of the generation of the tunnel sonic boom from a view point of gasdynamics.

\section{EXPERIMENTS}

Experiments were conducted by using a 1:250 train-tunnel simulator constructed in the Shock Wave Research Center, Tohoku University, and facility descriptions were reported by Sasoh et al. ${ }^{[6]}$ and Takayama et al. ${ }^{[9]}$. A brief introduction is given here for completeness. As shown in Fig.2, this train-tunnel simulator consists of a launch tube, an acceleration tube, a sabot separator, a tunnel section, a train model and a train model receiver. The train

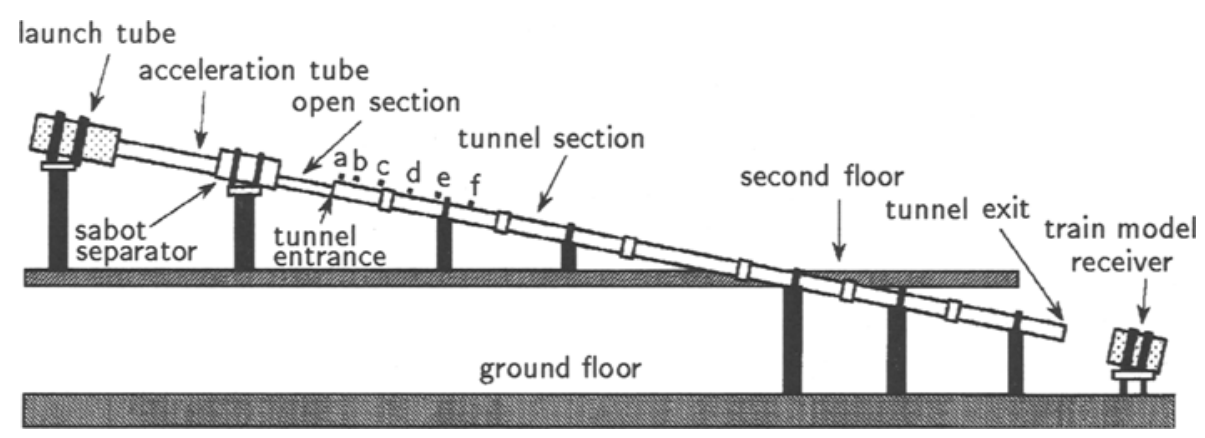

Fig.2 Experiment setup of a 1:250 scaled train-tunnel simulator in the Shock Wave Research Center, Tohoku University, Japan 
model, a plastic cylinder, is $18 \mathrm{~mm}$ in diameter and $40 \mathrm{~mm}$ in length. The tunnel section, functioning as a railway tunnel, is $40 \mathrm{~mm}$ in diameter and $25000 \mathrm{~mm}$ in length. So, the train-tunnel blockage ratio, the area ratio of the train model cross section to that of the tunnel section, is 0.20 . This value is widely accepted in the Japanese Shinkansen system.

In order to simulate tunnel sonic booms, two factors were considered in building up this train-tunnel simulator. The first one is the rapid deceleration of a train model due to friction from the direct contact between the steel tunnel section wall and the plastic train model when the train model is moving down the tunnel section. This leads to a weaker sonic boom at the exit of the tunnel section for a given train speed. To solve this problem, the train-tunnel simulator is inclined approximately at an angle of $8^{\circ}$ to the ground floor so that the gravitational force could be utilized to compensate the deceleration of the train model due to the friction. Calibration experiments demonstrated that the train speed was maintained almost constant while the train model moved throughout the tunnel section.

The other factor is how to simulate the abrupt-entering process of a high-speed train running into a railway tunnel. This was managed by installing an open section between the tunnel section and the acceleration tube. In order to guide the train model smoothly into the tunnel section, the train model is initially supported by $25 \mathrm{~mm}$ diameter sabots at both its frontal and rear end. The sabots are accelerated with the train model together along a $2 \mathrm{~m}$ long acceleration tube, but separated at a sabot separator section. This sabot separator also works to prevent the high pressure driver gas from overtaking the train model and blowing into the tunnel section. Performance tests about this train-tunnel simulator showing satisfactory results have been reported in detail by Takayama et al. ${ }^{[9]}$.

Calibrated pressure transducers (Kister 603B) are mounted at measurement stations

marked $\mathbf{a}, \mathbf{b}, \mathbf{c}, \mathbf{d}, \mathbf{e}$ and $\mathbf{f}$ along the tunnel section, as shown in Fig.2. The distance from the entrance to station $a$ is $40 \mathrm{~mm}$; that between stations $a$ and $b$ is $480 \mathrm{~mm}$; and the distance between other stations is $500 \mathrm{~mm}$. Pressure histories at these measurement stations are recorded on a transient recorder to verify appearance of shock waves, monitor the waves passing by and validate numerical results.

\section{NUMERICAL METHODS}

In order to simulate the four physical processes mentioned above, the length of a railway tunnel in a computational model must be taken to be, at least, 20 times longer than a high-speed train so that the expansion waves generated behind the train could propagate over a distance being long enough to overtake the train. Length of the train must be also long enough for the flow state transformation mentioned in the second wave process. Moreover, a free terrain must be added to the tunnel entrance in the computational domain to simulate the process of the train abrupt-entering into railway tunnels. This leads to a large scale ratio of a computational domain. Therefore, it is almost impossible to do truly three-dimensional numerical simulations even with super-computers because of complicated configurations of modern high-speed trains. Considering the scaled train-tunnel simulator introduced above, the flow motion in train-tunnel systems is modelled as an axisymmetric flow problem, which is a good approximation, at least, for the time being. The computational domain of the simplified train-tunnel system is shown in Fig.3, in which the train is simplified to a cylindrical piston and the tunnel section to a long constant cross-sectional tube. The cylindrical piston, 


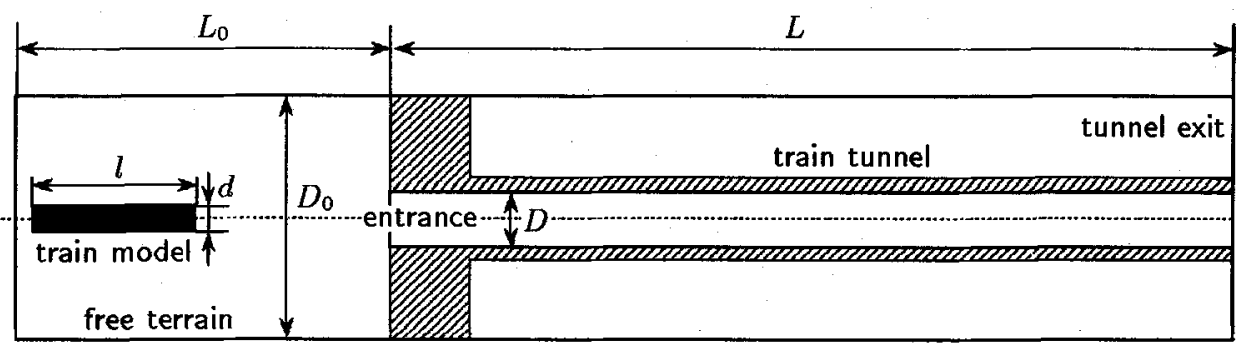

Fig.3 The simplified train-tunnel system for numerical simulations

called a train model here, moves at a given speed outside of the steel tube, called the tunnel section, then enters it and moves along its centerline. Parameters of the computational domain are given as follows: the train model diameter, $d$, is taken to be $18 \mathrm{~mm}$, and accepted as the reference length; the train model is $200 \mathrm{~mm}$ and $300 \mathrm{~mm}$ in length, respectively, for two cases. The tunnel section is $40 \mathrm{~mm}$ in diameter so that the train-tunnel blockage ratio could be 0.20 , the same as the scaled train-tunnel simulator. Tunnel section length, $L=6000 \mathrm{~mm}$; free terrain length, $L_{0}=1000 \mathrm{~mm}$; and the diameter of the free terrain, $D_{0}=100 \mathrm{~mm}$.

According to the experimental observation reported by Takayama et al. ${ }^{[9]}$, viscous effects on the wave propagation in a short tunnel section are so small that the Euler equations could be accepted as the governing equations for this train-tunnel flow motion.

\subsection{Governing Equations}

The hyperbolic system of the conservation laws for the perfect gas in cylindrical coordinates can be written as

$$
\frac{\partial \boldsymbol{U}}{\partial t}+\frac{\partial \boldsymbol{F}}{\partial x}+\frac{\partial \boldsymbol{G}}{\partial r}+\frac{1}{r} \boldsymbol{S}=0
$$

where $\boldsymbol{U}, \boldsymbol{F}, \boldsymbol{G}$ and $\boldsymbol{S}$ denote the state variable, flux and source, respectively, given by

$$
\boldsymbol{U}=\left(\begin{array}{c}
\rho \\
\rho u \\
\rho v \\
e
\end{array}\right) \quad \boldsymbol{F}=\left(\begin{array}{c}
\rho u \\
\rho u^{2}+p \\
\rho u v \\
(e+p) u
\end{array}\right) \quad \boldsymbol{G}=\left(\begin{array}{c}
\rho v \\
\rho u v \\
\rho v^{2}+p \\
(e+p) v
\end{array}\right) \quad \boldsymbol{S}=\left(\begin{array}{c}
\rho v \\
\rho u v \\
\rho v^{2} \\
(e+p) v
\end{array}\right)
$$

where primitive variables in the unknown $U$ are density $\rho$, velocity components $u$ and $v$ in $x$ - and $y$-direction, respectively, and total energy per unit volume $e . p=p(e, \rho)$ is the fluid pressure related to the equation of state for the perfect gas given as

$$
e=\frac{p}{\gamma-1}+1 / 2 \rho\left(u^{2}+v^{2}\right)
$$

where $\gamma$, the specific heat ratio, is taken as 1.4 in the present calculation.

\subsection{Numerical Methods}

The explicit finite difference equations of Eq.(1) discretized by using the second-order dispersion-controlled scheme ${ }^{[11]}$ are given in the form of a semi-discrete form as

$$
\left(\frac{\partial U}{\partial t}\right)_{i, j}^{n}=-\frac{1}{\Delta x}\left(\boldsymbol{H}_{i+1 / 2, j}^{n}-\boldsymbol{H}_{i-1 / 2, j}^{n}\right)-\frac{1}{\Delta r}\left(\boldsymbol{P}_{i, j+1 / 2}^{n}-\boldsymbol{P}_{i, j-1 / 2}^{n}\right)-\boldsymbol{S}_{i, j}^{n}
$$


with

$$
\begin{aligned}
& \boldsymbol{H}_{i+1 / 2, j}=\boldsymbol{F}_{i+1 / 2 L, j}^{+}+\boldsymbol{F}_{i+1 / 2 R, j}^{-} \\
& \boldsymbol{P}_{i, j+1 / 2}=\boldsymbol{G}_{i, j+1 / 2 L}^{+}+\boldsymbol{G}_{i, j+1 / 2 R}^{-} \\
& \boldsymbol{F}_{i+1 / 2 L, j}^{+}=\boldsymbol{F}_{i, j}^{+}+\boldsymbol{\Phi}_{\boldsymbol{A}}^{+} \operatorname{minmod}\left(\Delta \boldsymbol{F}_{i-1 / 2, j}^{+}, \Delta \boldsymbol{F}_{i+1 / 2, j}^{+}\right) / 2 \\
& \boldsymbol{F}_{i+1 / 2 R, j}^{-}=\boldsymbol{F}_{i+1, j}^{-}-\boldsymbol{\Phi}_{\boldsymbol{A}}^{-} \operatorname{minmod}\left(\Delta \boldsymbol{F}_{i+1 / 2, j}^{-}, \Delta \boldsymbol{F}_{i+\frac{3}{2}, j}^{-}\right) / 2 \\
& \boldsymbol{G}_{i, j+1 / 2 L}^{+}=\boldsymbol{G}_{i, j}^{+}+\boldsymbol{\Phi}_{\boldsymbol{B}}^{+} \operatorname{minmod}\left(\Delta \boldsymbol{G}_{i, j-1 / 2}^{+}, \Delta \boldsymbol{G}_{i, j+1 / 2}^{+}\right) / 2 \\
& \boldsymbol{G}_{i, j+1 / 2 R}^{-}=\boldsymbol{G}_{i, j+1}^{-}-\boldsymbol{\Phi}_{\boldsymbol{B}}^{-} \operatorname{minmod}\left(\Delta \boldsymbol{G}_{i, j+1 / 2}^{-}, \Delta \boldsymbol{G}_{i, j+\frac{3}{2}}^{-}\right) / 2 \\
& \Delta \boldsymbol{F}_{i+1 / 2, j}^{ \pm}=\boldsymbol{F}_{i+1, j}^{ \pm}-\boldsymbol{F}_{i, j}^{ \pm} \\
& \Delta \boldsymbol{G}_{i, j+1 / 2}^{ \pm}=\boldsymbol{G}_{i, j+1}^{ \pm}-\boldsymbol{G}_{i, j}^{ \pm} \\
& \boldsymbol{F}^{ \pm}=\boldsymbol{A}^{ \pm} \boldsymbol{U} \\
& \boldsymbol{G}^{ \pm}=\boldsymbol{B}^{ \pm} \boldsymbol{U} \\
& \boldsymbol{\Phi}_{\boldsymbol{A}}^{ \pm}=\boldsymbol{I} \mp \beta \boldsymbol{\Lambda}_{\boldsymbol{A}}^{ \pm} \\
& \boldsymbol{\Phi}_{\boldsymbol{B}}^{ \pm}=\boldsymbol{I} \mp \beta \boldsymbol{\Lambda}_{\boldsymbol{B}}^{ \pm}
\end{aligned}
$$

where $\boldsymbol{I}$ is a unit vector, $\beta=\Delta t / \Delta x$, and $\boldsymbol{\Lambda}_{\boldsymbol{A}}$ and $\boldsymbol{\Lambda}_{B}$ are vectors that consist of eigenvalues of matrices $\boldsymbol{A}$ and $\boldsymbol{B}$, respectively. The superscripts $(\cdot)^{+}$or $(\cdot)^{-}$denote vector flux splitting according to the Steger and Warming method ${ }^{[12]}$.

This scheme has been shown to meet the dispersion conditions ${ }^{[13,14]}$, therefore, shock waves could be captured without any numerical oscillation and the need of additional artificial viscosity. Such a characteristic is very helpful in highlighting week wave interactions, which otherwise may be easily diffused. The time-marching integration was performed by using a Runge-Kutta integration method of second-order accuracy. Reflection boundary conditions were used on the axis of symmetry and solid walls. Non-reflecting boundary conditions were applied to other boundaries. Calculations were carried out on the computational domain, as shown in Fig.3, with an equally-spaced grid of $100 \times 7000$ grid points.

\subsection{Moving Boundary Conditions}

Considering the complexity of the computational domain with a moving body, the multi-block approach is accepted in the present numerical simulation. The grid system consists of three blocks: the free terrain; the tunnel domain and the moving domain around the train model. In order to simulate a moving high-speed train, surfaces of the train model are traced step by step while it is moving in the fixed main mesh system so that the moving boundary conditions consistent with the Euler equations can be applied on the surfaces. A schematic diagram of the moving boundary condition is shown in Fig.4.

For example, to solve the governing equations at point $\mathbf{A}$, flow states at points $\mathbf{a}^{\prime}$ and $\mathbf{b}^{\prime}$ in the moving domain and within the boundary of the train model, are needed for our second-order scheme. Mirror images of these two points are placed at $\mathbf{a}$ and $\mathbf{b}$ in the main domain. This means that distances $a w=w a^{\prime}$ and $b w=w b^{\prime}$, and these two lines are normal to the surfaces. At each time step, coordinates of points $\mathbf{a}$ and $\mathbf{b}$ are first calculated according to the moving surface, and then interpolation is applied to get flow states at these 


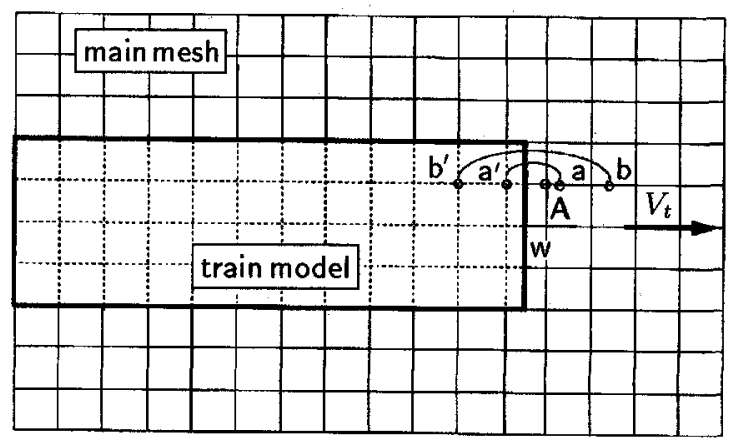

Fig.4 Schematic diagram of moving boundary conditions on a train model

points. Finally, "mirror-image" flow states at virtual points in the moving domain are specified according to the law of the moving slip-boundary condition, for example, numerical formula at point $\mathbf{a}^{\prime}$ are given as follows

$$
\begin{aligned}
& u_{\mathrm{a}^{\prime}}=2.0 V_{t}-u_{\mathrm{a}} \\
& v_{\mathrm{a}^{\prime}}=v_{\mathrm{a}} \\
& \rho_{\mathrm{a}^{\prime}}=\rho_{\mathrm{a}} \\
& p_{\mathrm{a}^{\prime}}=p_{\mathrm{a}}
\end{aligned}
$$

where $V_{t}$ is the train speed. Thus, the surface of the train model behaves like a moving solid wall. The number of interpolated points needed depends on the accuracy order of the numerical scheme. The boundary condition has been carefully verified by Jiang et al. ${ }^{[14]}$.

\section{COMPARISON BETWEEN NUMERICAL AND EXPERIMENTAL RESULTS}

Results of two cases having the same train length of $200 \mathrm{~mm}$ are presented in this section. The train speed is taken to be $360 \mathrm{~km} / \mathrm{h}$ in the first case and $270 \mathrm{~km} / \mathrm{h}$ in the second. Other parameters are the same as described in the computational domain. In the two cases, the train model was accelerated from zero to a specified speed outside of the tunnel section at first, and then moves into the tunnel section with a constant speed. This is to minimize the impulse pressure rise induced due to the abrupt-starting of the train near the entrance. The speed of the train model is maintained constant throughout the tunnel section.

Figure 5 shows numerical pressure histories simulated at three measurement stations marked with $\mathbf{a}, \mathbf{c}$ and $\mathbf{d}$ in the first case. The corresponding experimental results measured at those stations are presented in Fig.6. From those two figures, some common features are observable. The first feature is an impulse pressure rise in front of a column of air flow with uniform pressure, and this impulse pressure rise develops from compression waves generated at the entrance of the tunnel section. The second feature is a uniform column of the flow behind the impulse pressure rise and the column length varies with the position where pressure transducers are mounted. This is because the compression waves ahead of the train propagate at the local sound speed that is much greater than the train speed. The last feature is the sharp decrease of the flow pressure that follows the uniform flow, which is recognized to be due to pressure variations when the train passes the measurement 


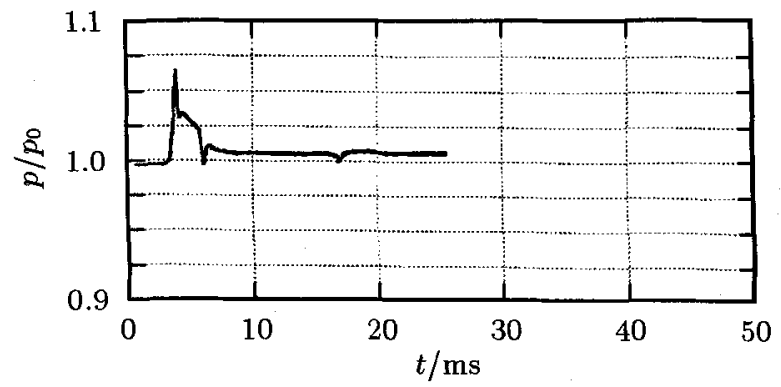

(a)

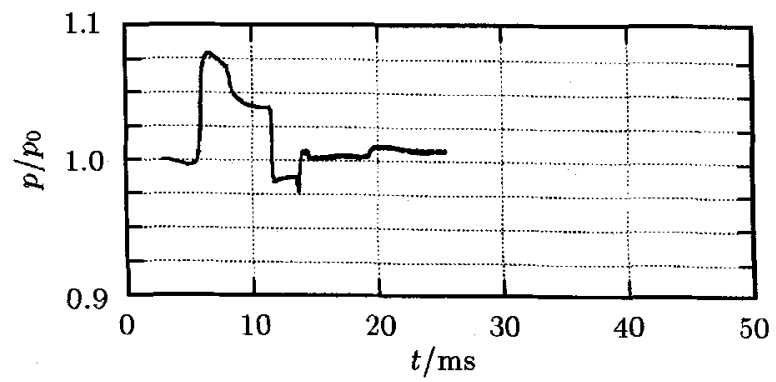

(b)

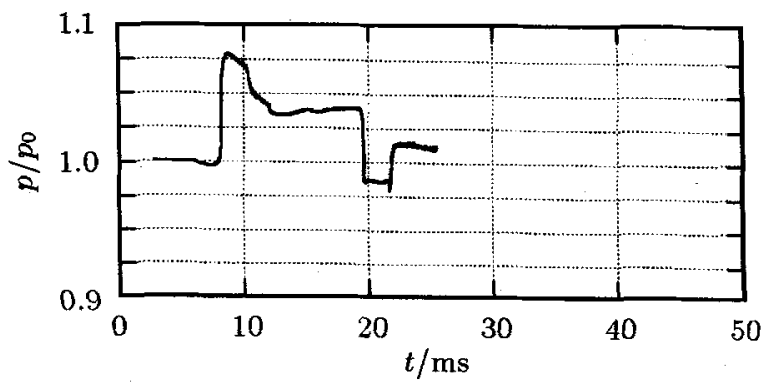

(c)

Fig.5 Numerical pressure histories simulated at stations a, c and $\mathbf{d}$ for $V_{t}=360 \mathrm{~km} / \mathrm{h}$ and $l=200 \mathrm{~mm}$

stations. So, those numerical and experimental pressure profiles are in good agreement and show the same wave characteristics in the train-tunnel problem. It could mean that the viscosity effect on the wave dynamic process is not significant so that the flowfield could be well described by the Euler equations. Moreover, the wave propagating speed is much higher than the train speed so that the wave process could be completed very rapidly. Therefore, effects on the wave process are small whether the train moves along the centerline or the bottom of the tunnel section.

The experimental results show that the maximum overpressure in the impulse pressure rise is about $7.5 \mathrm{kPa}$ and the average pressure in the uniform column of air is $2.1 \mathrm{kPa}$. As to the numerical results, the maximum overpressure is about $7.5 \mathrm{kPa}$ and the average pressure is about $3.0 \mathrm{kPa}$. Hence, the maximum overpressure agrees well, but the average pressure is over-estimated in the numerical solutions. Considering that numerical simulations are for 


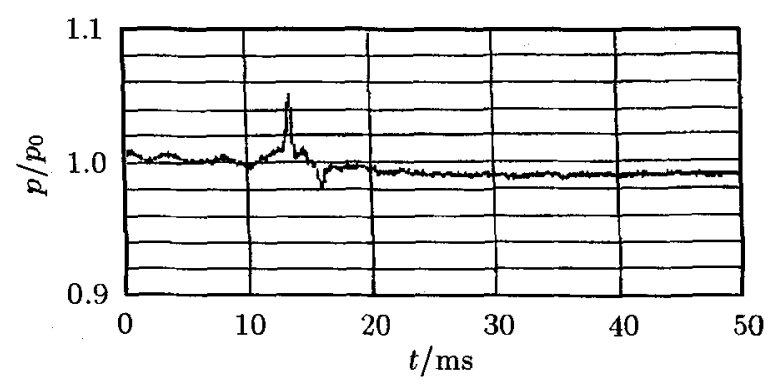

(a)

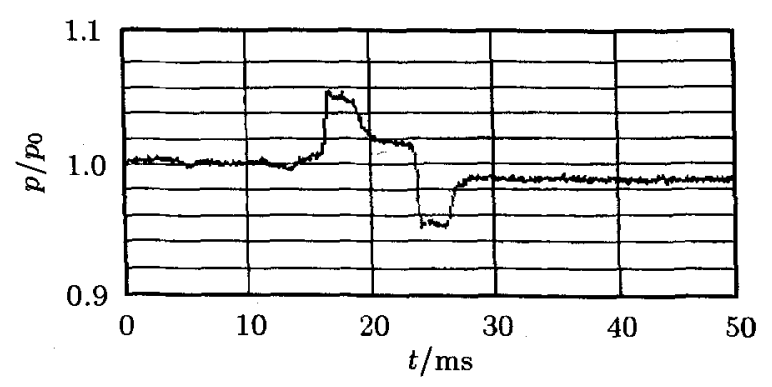

(b)

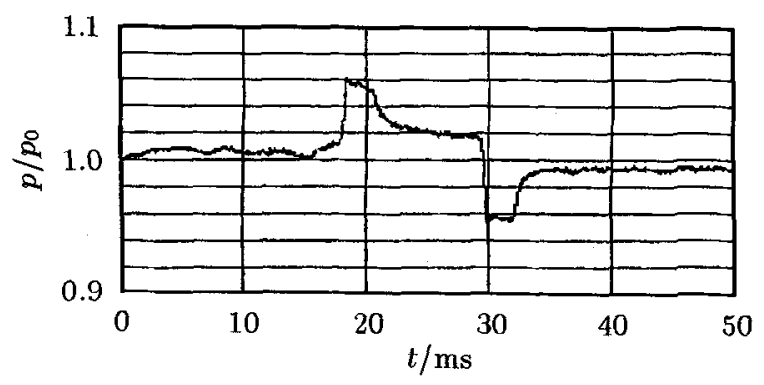

(c)

Fig.6 Experimental pressure histories measured at stations a, c and $\mathrm{d}$ for $V_{t}=360 \mathrm{~km} / \mathrm{h}$ and $l=200 \mathrm{~mm}$

inviscid cases, the above validation result is reasonable since the maximum pressure is mainly determined by wave processes and the average pressure is affected by viscosity and turbulence. The more important factor is that the good agreement between Figs.5 and 6 means that the numerical simulations do reproduce the wave processes occurring in the experiments, which are intended to do in this paper.

By carefully examining the impulse pressure rise, it is seen that the impulse pressure rise consists of three parts having different overpressure values. The first part is the peak pressure created due to the abrupt-reduced space around the train model while the head of the train model moves into the entrance of the tunnel section. The second one is the gradually-decreasing overpressure behind the sharp pressure jump, which is generated due to the flow state transition from the abrupt-entering flow state to the one resulting from an infinitely long train model that moves into the tunnel section. The last one is the quicklydecreasing overpressure due to the fact that expansion waves generated behind the train 
model overtake it.

The uniform part of the overpressure behind the impulse pressure rise develops while the train model moves in the tunnel section. During this period, the flowfield around the train model looks like a steady state flow if observed from the train model. The reason is that the constant drag force acting on the train model provides energy and momentum for the uniform column of air ahead of the train model. This phenomenon is consistent with the conservation laws of momentum and energy, and also is self-sustained.

Numerical and experimental pressure histories recorded at three stations a, c and $\mathbf{d}$ for the second case are shown in Figs.7 and 8, respectively. The experiment of this case is carried out by using the same train-tunnel configuration as the first case but for a train speed of $270 \mathrm{~km} / \mathrm{h}$ to examine the role of train speed and avoid "coincidence" in CFD validation. From these results, the same agreement between the numerical results and the experiments

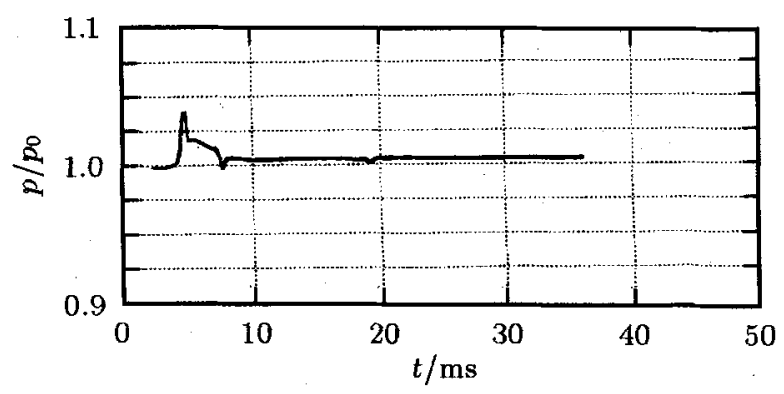

(a)

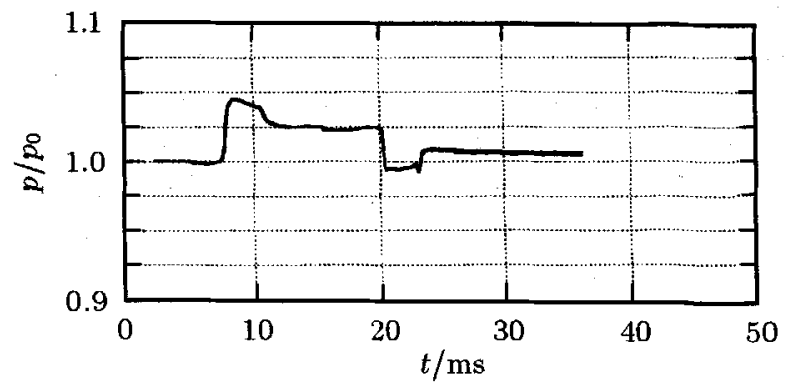

(b)

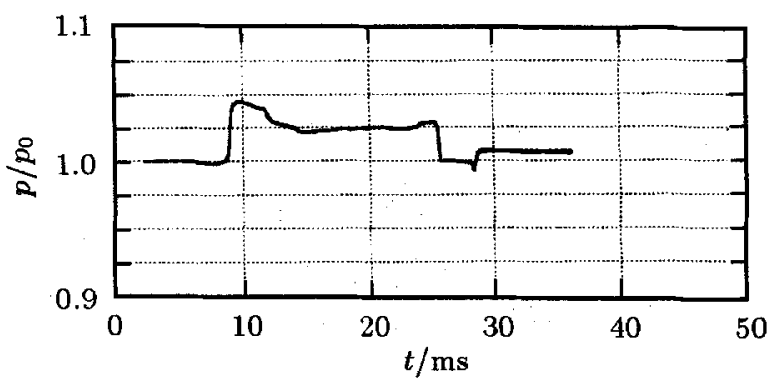

(c)

Fig.7 Numerical pressure histories simulated at stations a, $c$ and $\mathrm{d}$ for $V_{t}=270 \mathrm{~km} / \mathrm{h}$ and $l=200 \mathrm{~mm}$ 


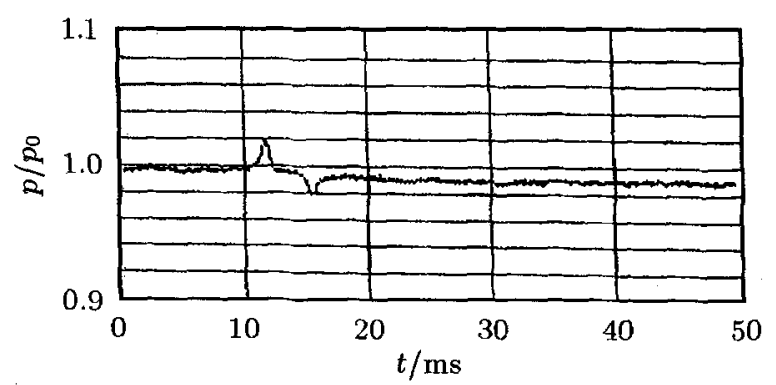

(a)

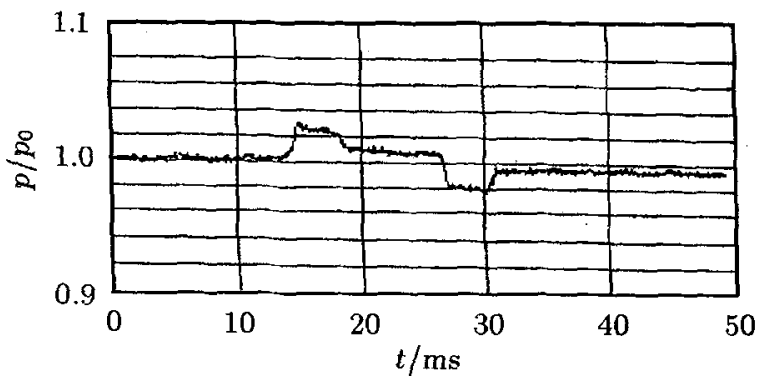

(b)

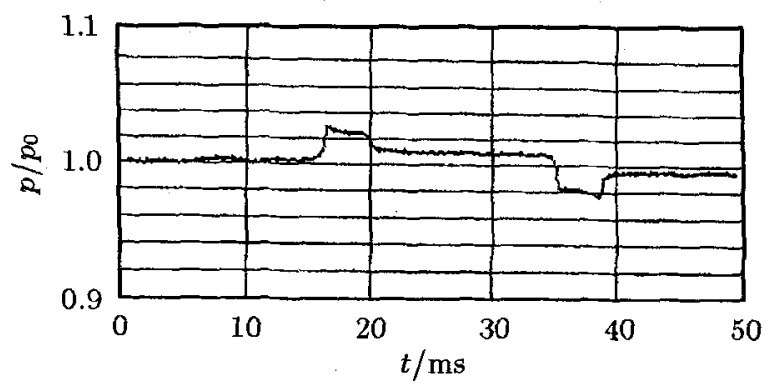

(c)

Fig.8 Experimental pressure histories measured at stations a, $c$ and $\mathrm{d}$ for $V_{t}=270 \mathrm{~km} / \mathrm{h}$ and $l=200 \mathrm{~mm}$

could be demonstrated, and the CFD validation is confirmed again. The only discrepancy observed from these two cases is that the maximum overpressure of the impulse signs in the second case is less than $5 \mathrm{kPa}$. This value is $50 \%$ lower than the first case but the train speed is only $25 \%$ lower. This implies that the overpressure of tunnel sonic booms will increase dramatically against train speed and the reduction of tunnel sonic booms is significant for new high-speed train systems.

\section{GENERATION AND PROPAGATION OF WAVES IN A RAILWAY TUNNEL}

In order to interpret the pressure histories presented in Fig.5, a time sequence of isobars of the train-tunnel flow is shown in Fig.9. The corresponding pressure profiles along the axis of symmetry at each stage are plotted in Fig.10. From those results, a detailed description on wave dynamic processes in the tunnel section is given as follows. Figure 9(a) shows the 


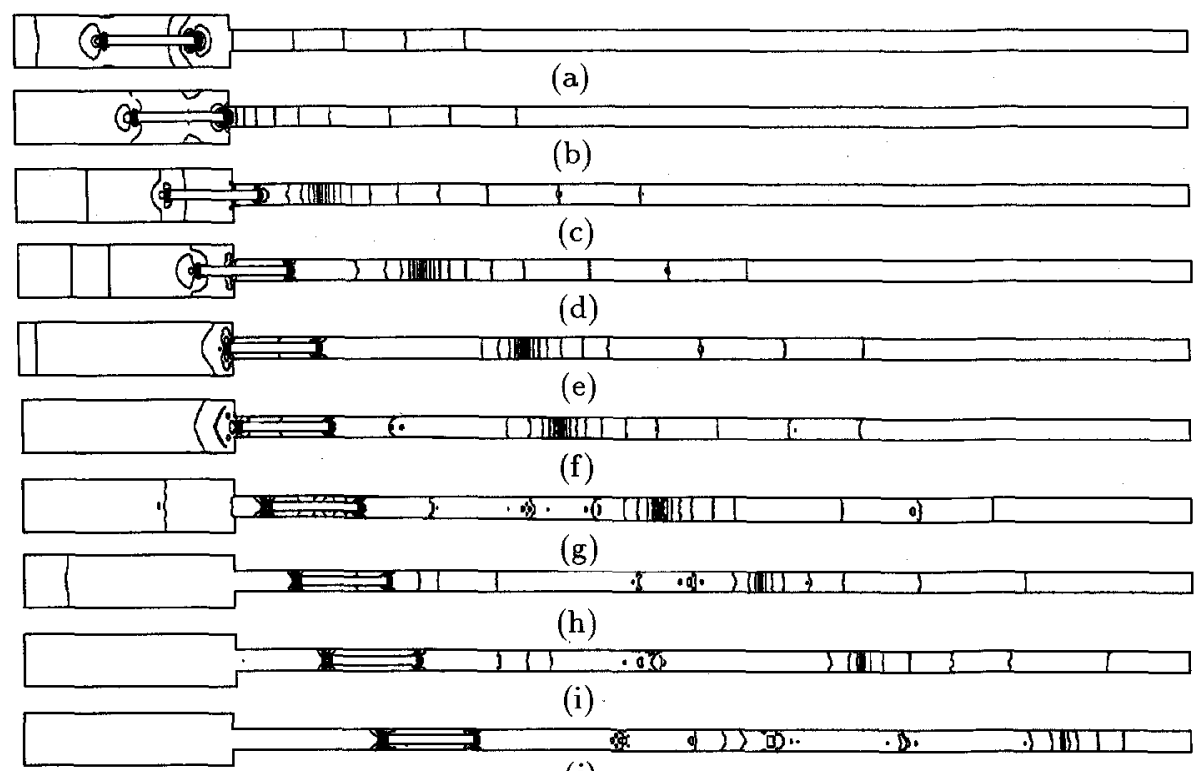

(j)

Fig.9 A time sequence of pressure distributions of the flowfield for $V_{t}=360 \mathrm{~km} / \mathrm{h}$ and $l=200 \mathrm{~mm}$

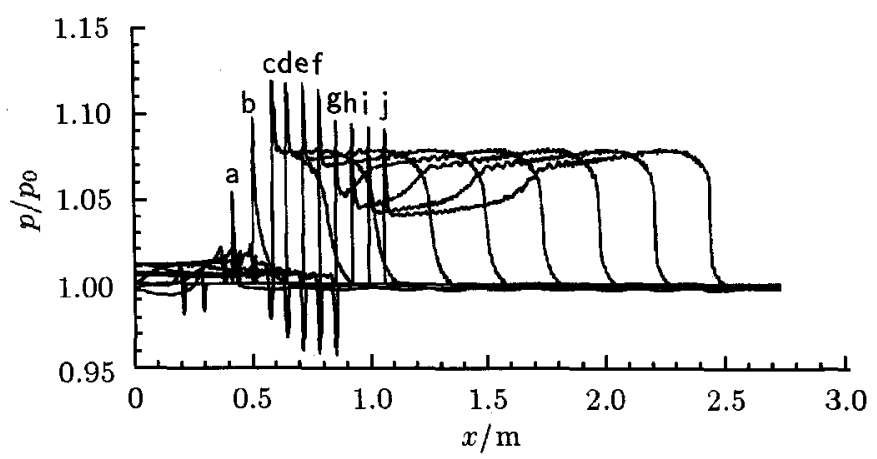

Fig.10 A time sequence of pressure profiles along the axis of the tunnel section centerline for $V_{t}=360 \mathrm{~km} / \mathrm{h}$ and $l=200 \mathrm{~mm}$

result at the initial moment before the train model enters the tunnel. The initial position of the front of the train model is set at a distance of twice of the train model length away from the entrance. No impulse pressure rise is observable in the tunnel section, but very weak pressure perturbations (a few isobars in the tunnel) still move into it. This is acceptable because the sound waves would first propagate into a train tunnel in a real case while a train is approaching the entrance. Stagnation pressure shown in the pressure profile marked with $\mathrm{a}$ in Fig. 10 has a value of about $5.85 \mathrm{kPa}$. The value is approximately equivalent to the stagnation pressure calculated for the train speed of $360 \mathrm{~km} / \mathrm{h}(100 \mathrm{~m} / \mathrm{s})$. This pressure profile looks similar to the case where a train moves over a free terrain. The diameter of the train-affected area is estimated to be about three times larger than the train model diameter. 
Figures $9(\mathrm{~b})$ and $9(\mathrm{c})$ show the flowfield while the head of the train model is moving into the tunnel section. A series of compression waves are generated in front of the train model due to the abrupt space contraction around the train model at the entrance. These waves are initially curved, but develop into planar ones very quickly due to repeated reflections from tunnel section walls as shown in Fig.9(b). This implies that the effect of the frontal shape of the train on wave propagations is small, but may be significantly different on drag force. The compression waves propagate forward at local sound speed so that the high pressure region in front of the train model could become longer and longer as shown in Figs.9(d) and 9(e). By carefully examining the compression wave structures shown from Figs.9(c) to 9(i), the isobars within the wave front become denser and denser, and then, due to the wave coalescence, a shock wave is built up gradually in Fig.9(j) as reported by Takayama et al. ${ }^{[9]}$. This is a typical feature of non-linear property of compression wave propagation, which cannot be well treated with acoustic theory.

From the pressure profiles marked with $\mathbf{b}, \mathbf{c}$ and $\mathbf{d}$ in Fig.10, the stagnation pressure increases drastically upon the abrupt-entering of the train model into the tunnel section and then decreases with further motion of the train model into the tunnel section, as shown by the pressure profiles marked with $\mathbf{e}, \mathbf{f}$ and $\mathbf{g}$. The maximum stagnation pressure reached is twice higher than that created by the train model moving over a free terrain. This phenomenon.agrees with the observation that the aerodynamic drag force acting on the train increases dramatically once the train moves into a railway tunnel, similar to the wave resistance occurring in the supersonic aircraft.

Flow transition from the abrupt-entering flow state to that resulting from an infinitely long train model moving into the tunnel section is clearly observed from the pressure profiles marked with $\mathbf{d}$, $\mathbf{e}$ and $\mathbf{f}$ as shown in Fig.10 where the overpressure behind the wave front decreases gradually due to changes of train-affected area.

Figures $9(\mathrm{f})$ and $9(\mathrm{~g})$ show that a series of expansion waves were generated behind the train model due to the abrupt-entering of the rear end of the train model. These waves propagate both forward and backward at the local sound speed. The forward-propagating waves catch up with the train model as shown in Fig.9(g) and then overtake it as shown in Figs. 9(h) to 9(i). These expansion waves decrease the overpressure of the air flow in front of the train model as shown by the pressure profiles marked with $\mathbf{f}$ and $\mathbf{g}$ in Fig.10 where the rapid decrease in the overpressure behind the wave font is readily observable.

Constant pressure behind the expansion waves is observed in Figs.9(i) and 9(j). The air flow around the train model seems to reach a steady state if observed in the trainfixed coordinates. In the pressure profile marked with $\mathbf{j}$ in Fig.10, a uniform pressure area behind the expansion waves is observed. This phenomenon is similar to the case of a piston moving in a tube, in front of which a column of gases with uniform pressure is built up. However, in the train-tunnel problem, the phenomenon appears to be more complex due to the interaction between expansion and compression waves.

\section{EFFECTS OF TRAIN-TUNNEL CONFIGURATIONS}

In order to examine the effect of train length, two pressure profiles simulated at station e are shown in Fig.11 for the train speed of $360 \mathrm{~km} / \mathrm{h}$. Figure 11(a) shows the case of a $200 \mathrm{~mm}$ train model. Figure 11(b) shows the case of a train model of $300 \mathrm{~mm}$ long. Basically 


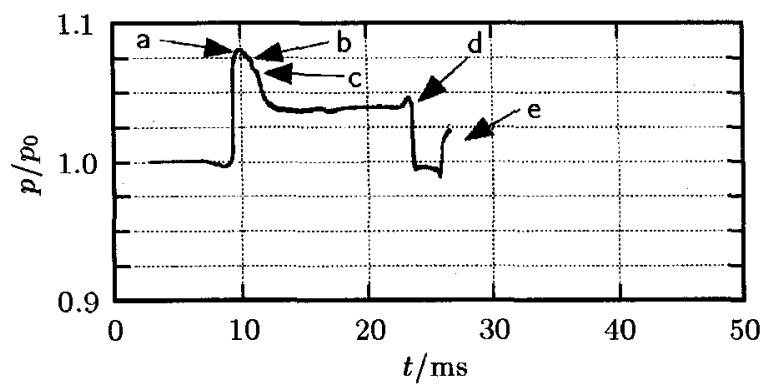

(a) $l=200 \mathrm{~mm}$

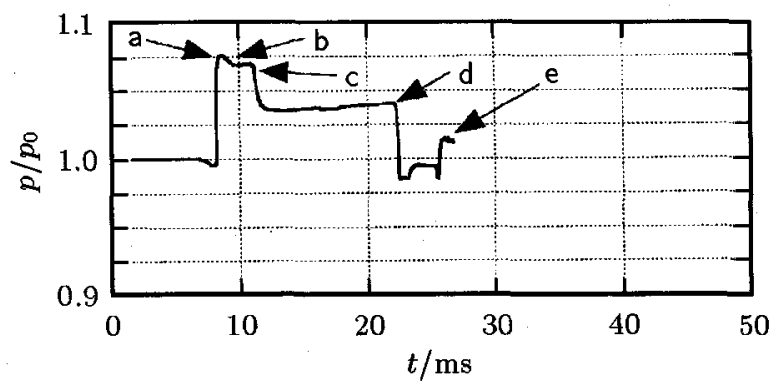

(b) $l=300 \mathrm{~mm}$

Fig.11 Pressure histories at station e for $V_{t}=360 \mathrm{~km} / \mathrm{h}$

speaking, those two pressure profiles look similar to each other. The only discrepancy observable from these profiles is the flow transition from the abrupt-entering flow state to the one resulting from an infinitely long train model moving into a train tunnel. This process is clearly shown in Fig.11(b) where a constant pressure region from positions $\mathbf{b}$ to $\mathbf{c}$ behind the wave front exists. However, it is not easy to identify that region from Fig.11(a) because the expansion waves overtake the train model just at the moment when the flow transition is completed. For the train-tunnel experiments, the train model length must be taken to be, at least, 15 times longer than its diameter to observe this flow transition. In the real case, trains are so long that this would not be a problem.

To gain a better understanding on overpressure variations resulting from wave interactions, the pressure profiles in Fig.11 are examined in detail as follows: The pressure jump marked with $\mathbf{a}$ is created by the shock wave which develops from the precursor compression waves generated at the entrance due to the sudden space contraction around the train model. This maximum overpressure is very difficult to be estimated because the flowfield near the entrance is highly transient and non-linear. However, it is easy to understand that the overpressure depends on both the shape of the train model and tunnel entrance configurations. The cone-shaped train head and the hood installed at the tunnel entrance both are helpful to reduce this overpressure ${ }^{[2,9]}$. Such train-tunnel configurations gradually reduce the space around the train at the entrance.

Position marked with $\mathbf{b}$ in Fig.11(b) is the point at which the flow transition to the one related to an infinitely long train model moving into a train tunnel is completed. From point $\mathbf{c}$, the expansion waves generated behind the train model have overtaken the train model so that the fluid pressure in front of the train is decreased to a constant level. The 
time duration from points a to c depends on the train model length and train speed. The longer the train is, the longer the distance for a given train speed. The overpressure between points $\mathbf{b}$ and $\mathbf{c}$ is equal to that induced by an infinite long train model moving into the tunnel section without considering the entrance effect. Some theoretical formula are based on this stage ${ }^{[1 \sim 3]}$ because both the entrance effect and expansion-compression wave interactions are very difficult to be modeled. Therefore, in comparing experimental results with theoretical ones, more reasonable conclusions may be obtained if carefully choosing overpressure in experimental pressure histories. As is well known, a sharp pressure that is not self-sustained is easy to attenuate, therefore, the level of tunnel sonic booms depends on this overpressure for a train tunnel that is long enough for compression waves to transform into a weak shock wave. Considering the short duration time of the overpressure, the hood installed at the tunnel exit is also helpful for the noise reduction from the view point of wave diffraction.

The constant pressure between points $\mathbf{c}$ and $\mathbf{d}$ corresponds to the flow state due to the train model moving in the tunnel section after the expansion waves behind the train model have overtaken it, and before the expansion waves induced by the shock wave discharge come back. The length of the column of air can be easily calculated if the tunnel length and train speed are given with the assumption that both expansion and compression waves propagate at sound speed. However, this constant overpressure is quite difficult to be estimated because effects of expansion waves are unknown. It may not be important for certain train tunnels because this overpressure will be decreased by the up-stream running expansion waves generated at the exit after the weak shock wave discharges. However, for a very long train tunnel where wave attenuation will function significantly, the maximum overpressure of the impulse will attenuate proportionally against the distance to this overpressure that is related to a self-sustained wave process. The real tunnel experiments reported by Ozawa ${ }^{[2]}$ and Takayama ${ }^{[9]}$ for real train tunnels (several kilometers long) showed only a long duration overpressure without any impulse pressure rise. In such a case, the level of tunnel sonic booms depends on this overpressure and perforated walls lined inside train tunnels may work for overpressure reduction.

In addition, point $\mathbf{d}$ indicates that the head of the train model reaches the measurement station, and point $\mathbf{e}$ implies that the rear of the train model passes the station. This low pressure means that the particle velocity beside the train model is higher and the flow pressure becomes lower. This particle velocity is related to vortex motions beside trains, which is another important topic in the train-tunnel dynamics.

The train-tunnel blockage ratio is also a major parameter closely related to tunnel sonic booms. The smaller the train-tunnel blockage ratio is, the weaker the shock wave thus generated. However, the small train-tunnel blockage ratio means a huge expense for railway tunnel constructions.

Figure 12 shows the overpressure against the train-tunnel blockage ratio for the train speed of $360 \mathrm{~km} / \mathrm{h}$. The solid line is plotted from the result calculated with the formula proposed by Hara ${ }^{[1]}$. As to the numerical results, simulations are carried out by varying the tunnel diameter. The average overpressure in the impulse pressure rise is shown with triangle symbols and that in the uniform area behind the impulse pressure rise is denoted by square symbols. In two extreme cases, these two overpressures are the same. For instance, when the train-tunnel blockage ratio is zero the overpressure approaches to zero, too. If the blockage ratio is 1.0 , this overpressure becomes the post-shock overpressure of the shock 


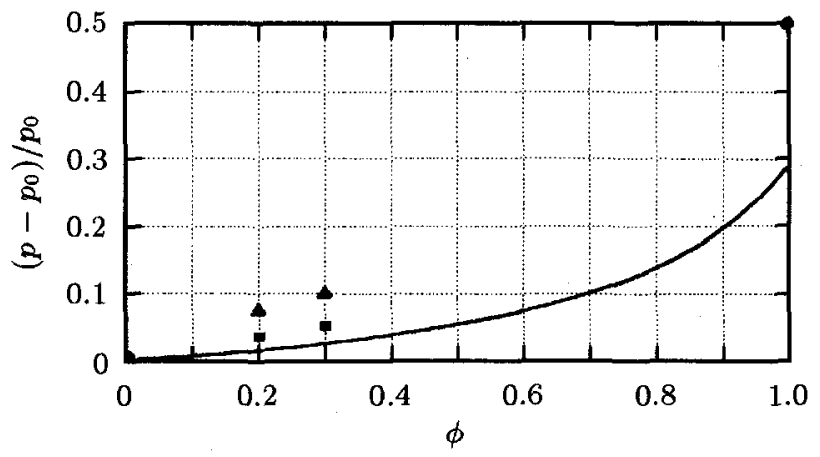

Fig.12 Overpressure against the train-tunnel blockage ratio for $V_{t}=360 \mathrm{~km} / \mathrm{h}$

wave having the post-shock particle velocity equal to the train speed.

From Fig.12, it is also observed that the overpressure nonlinearly rises against the train-tunnel blockage ratio and numerical results (the overpressure in the uniform area) are about $60 \%$ higher than that predicted with acoustic theory. Therefore, non-linearity of wave propagation in the high-speed train-tunnel system is significant. Moreover, it is worth pointing out that the overpressure presented here is obtained at the position near the tunnel entrance and depends only on train-tunnel configurations and train speed. In other words, wave attenuation and viscosity effects are not considered here. When comparing these results with experimental results obtained at positions several kilometers away from the tunnel entrance, the wave attenuation must be considered because the overpressure decreases linearly against distance, as reported by Takayama ${ }^{[9]}$.

\section{CONCLUSIONS}

Aiming at a better understanding of tunnel sonic booms, the wave propagation and interaction in train-tunnel systems are simulated experimentally by using a scaled train-tunnel simulator, and numerically by solving the Euler equations based on the dispersion-controlled scheme combined with moving boundary conditions. The good agreement achieved between the experiments and the numerical simulations confirms four wave dynamic processes. Both numerical and experimental results show that the overpressure in the train-tunnel problem consists of two parts: The first part is an impulse pressure rise due to the abrupt-entering of the train head and the other part of constant overpressure develops when the expansion waves overtake the train. For a given train speed, the impulse pressure rise is related to train-tunnel configurations. The uniform pressure depends mainly on the train-tunnel blockage ratio. Resulting from nonlinear wave interaction, two overpressures are difficult to be predicted by conventional acoustic theory. They contribute to the tunnel sonic booms in different ways according to the tunnel length.

\section{REFERENCES}

1 Hara T. Aerodynamical problems when train is running into tunnel with large velocity. Railway Technical Research Report, the Railway Technical Research Institute, Japan, 1960. 61 (in Japanese) 
2 Ozawa S. Investigation of pressure wave radiated from a tunnel exit. Railway Technical Research Report, the Railway Technical Research Institute, Japan, 1976. 1023 (in Japanese)

3 Ozawa S. Studies of micro-pressure wave radiated from a tunnel exit. Railway Technical Research Report, the Railway Technical Research Institute, Japan, 1979. 1121 (in Japanese)

4 Sajben M. Fluid mechanics of train-tunnel systems in unsteady motion. AIAA Journal, 1971, 9: $1538 \sim 1645$

5 Matsuo K, Aoki T. Wave problems in high-speed railway tunnels. In: Takayama K ed. Proc of the 18th Int Symp on Shock Waves, Sendai, Japan, 1991-7-21 26. Berlin: Springer Verlag, 1991. $95 \sim 102$

6 Sasoh A, Onodera O, Takayama K, et al. Experimental investigation on the reduction of railway tunnel sonic boom. Jpn Soc Mech Eng, 1994, 580: 4112 4118

7 Kage K, Miyake H, Kawagoe S. Numerical study of compression waves produced by high-speed trains entering a tunnel. JSME Int Journal, Series B, 1995, 38: 191 198

8 Ogawa T, Fujii K. Numerical simulation of compressible flows induced by a train moving into a tunnel. Comp Fluid Dynamics J, 1994, 3: 63 82

9 Takayama K, Sasoh A, Onodera O, et al. Experimental investigation on tunnel sonic boom. Shock Waves, 1995, 5: 127 138

10 Sasoh A, Matsuoka K, Nakashio K, et al. Attenuation of weak shock waves along pseudoperforated walls. Shock Waves, 1998, 8: 161 172

11 Jiang Z. Study on the finite difference theory and numerical methods of weak solution problems. [Dissertation], Peking University, Beijing, China, 1993

12 Steger JL, Warming RF. Flux vector splitting of the inviscid gasdynamic equations with applications to finite difference methods. J Comp Phys, 1981, 40: 263 293

13 Zhang HX, Zhuang FG. NND schemes and its applications to numerical simulation of two and three dimensional flows. In: Proc of the 4th Int Symposium of CFD, Nagoya, Japan, 1998-0828 31, 1989. 149 152

14 Jiang Z, Takayama K, Chen YS. Dispersion conditions for non-oscillatory shock capturing schemes and its applications. Computational Fluid Dynamics J, 1995, 4: 137 150

15 Jiang Z, Takayama K, Skews BW. Numerical study on blast flowfields induced by supersonic projectiles discharged from shock tubes. Phys Fluids, 1998, 10(1): 277 288 